\title{
Lusioersily
}

\section{Reliable object handover through tactile force sensing and effort control in the Shadow Robot hand}

Gomez-Eguiluz, A., Rano, I., Coleman, S., \& McGinnity, T. M. (Accepted/In press). Reliable object handover through tactile force sensing and effort control in the Shadow Robot hand. In Unknown Host Publication IEEE. http://uir.ulster.ac.uk/37134/2/Decision\%20on\%20ICRA\%202017\%20submission\%201016.eml

Link to publication record in Ulster University Research Portal

\section{Published in:}

Unknown Host Publication

Publication Status:

Accepted/In press: 15/01/2017

\section{Document Version \\ Author Accepted version}

\section{General rights}

Copyright for the publications made accessible via Ulster University's Research Portal is retained by the author(s) and / or other copyright owners and it is a condition of accessing these publications that users recognise and abide by the legal requirements associated with these rights.

\section{Take down policy}

The Research Portal is Ulster University's institutional repository that provides access to Ulster's research outputs. Every effort has been made to ensure that content in the Research Portal does not infringe any person's rights, or applicable UK laws. If you discover content in the Research Portal that you believe breaches copyright or violates any law, please contact pure-support@ulster.ac.uk. 


\title{
Reliable object handover through tactile force sensing and effort control in the Shadow Robot hand
}

\author{
A. Gómez Eguíluz ${ }^{1}$, I. Rañó ${ }^{1}$, S.A. Coleman ${ }^{1}$ and McGinnity ${ }^{2}$ \\ ${ }^{1}$ Intelligent Systems Research Centre, Ulster University, Northern \\ Ireland, UK \\ ${ }^{2}$ School of Science \& Technology, Nottingham Trent University, UK
}

(C) 2017 IEEE. Personal use of this material is permitted. Permission from IEEE must be obtained for all other uses, in any current or future media, including reprinting/republishing this material for advertising or promotional purposes, creating new collective works, for resale or redistribution to servers or lists, or reuse of any copyrighted component of this work in other works. 


\title{
Reliable object handover through tactile force sensing and effort control in the Shadow Robot hand
}

\author{
A. Gómez Eguíluz ${ }^{1}$ and I. Rañó ${ }^{1}$ and S.A. Coleman ${ }^{1}$ and T.M. McGinnity ${ }^{2}$
}

\begin{abstract}
A fundamental problem in cooperative HumanRobot Interaction is object handover. Existing works in this area assume the human can reliably grasp the object from the robot hand. However, in some situations the human can produce perturbing forces in the object that are not meant to end in a handover. These perturbations can result in the object being dropped or the robot hand being damaged. This paper addresses this problem and presents a mechanism for reliable robot to human object handover implemented in a Shadow Robot hand endowed with tactile sensing. Given a stable grasping configuration, using BioTAC sensors we are able to estimate the contact forces applied to the object, and provide a feedback signal to a joint effort controller to maintain grasp forces despite perturbations. Our system is able to identify between object pulling forces which should result in an object handover, and other disturbances. Experimental results show that the hand releases the object only when the object is pulled, validating the proposed algorithm.
\end{abstract}

\section{INTRODUCTION}

Safe and smooth human-robot collaboration attracts huge research efforts in robotics. Joint manipulation and object hand-over are among the most important aspects of robots working alongside humans. These are complex, research areas; on their own since, for instance, human-robot object handover involves multiple aspects such as human and object safety, social and handling context, grasping stability and slip detection, and ergonomics. For example humans prefer certain handover configurations [1], but this preference is affected by the intended use of the object the robot is handing over [2]. In some complex situations, for instance in a mechanical workshop, the handover must be reliable as an operator might not be able to grasp an object in a fully secure way. This paper proposes a reliable object handover mechanism using tactile sensing and effort control, to ensure the person is ready to hold the object, avoiding damage for both the object and the robot itself.

Object handover is closely related to grasp stability, as the robot hand holding the object has to keep it stable until the human is ready to hold it. This is in fact the approach taken in one of the pioneering works on human-robot object exchange [3]. The implementation of a grasping system and the evaluation of (different forms of) grasp stability was used to control a three fingered robotic hand to hold and release objects when interacting with a human. A combination of joint angles,

\footnotetext{
${ }^{1}$ A. Gómez Eguíluz, I. Rañó, and S.A. Coleman are with the Intelligent Systems Research Centre, Ulster University, Northern Ireland, UK Gomez_Eguiluz-A@email.ulster.ac.uk \{i.rano, sa.coleman\}eulster.ac.uk

2 T.M. Mcginnity is with the School of Science \& Technology, Nottingham Trent University, UK martin.mcginnityentu.ac.uk
}

contact, kinematic, and dynamic stability indexes was used by the hand to decide whether to open the fingers (releasing the object) to close them (holding it), or to re-adjust one of the fingers in case contact was lost. While some handover research focuses on finding appropriate configurations to make the task easier for humans, conversely [4] shows that humans adapt the way they hand objects over to the specific configuration of the robot hand, simplifying the grasping problem. In their work the velocity of the hand is used to detect when the robot had to open or close the hand in order to release or grasp the object respectively during the interaction of the robot and the human.

As a part of a much larger project to build a butler robot, [5] implements a drink handover mechanism. Because of the potential troubles dropping a drink can cause, the robot in this case opened the gripper only when a human face was detected by the robot and, simultaneously, the person was pulling from the beverage displacing the compliant hand of a PR2 by one centimetre in the vertical direction. The first effort to imitate the actual way humans handover objects is presented in [6]. Their experiments showed that humans adapt the grasping force according to the change in the estimated weight of the object. Using a two finger hand and force sensors, the authors identify the slippage of the object, i.e. the tipping point on the Coulomb force, to release the object. Based on similar results about human handover [7], the work in [8] presents an object release controller implemented on a PR2 robot. The grip force of the robot is controlled according to the load force the robot perceives in the wrist. The authors perform a comparison with other handover controllers for quick release and constant grip forces. A user evaluation reveals that the participants' preference was the controller imitating human handover, as it produced less likelihood of the object dropping and the object was easier to accept by humans.

Existing works in object handover assume the handover is going to take place with no potential problem, although it might not be always the case. Therefore, in some situations the robot should not release the object, for instance if the force over the object does not have the right direction. If the human pushes or rotates the object unintentionally it might result in the object dropping. On the other hand, if the perturbing force is too high the robot hand could be damaged, hence the robot must adapt its configuration to try and keep holding the object. This paper contributes a solution to this problem by proposing a reliable object handover algorithm implemented in a Shadow Robot Hand through force sensing and a joint effort controller. The rest of the paper is organised 
as follows. First, Section II presents the effort controller and force estimation procedures, followed by the reliable object handover algorithm to keep the object grasped or release it according to the force applied to the object. Section III shows results on the adaptation of the hand fingers when different forces are applied, and on the release algorithm. Finally Section IV presents conclusions and directions for future work.

\section{RELIABLE OBJECT HANDOVER CONTROLLER}

Reliable robot to human object handover requires a system that can provide adaptability against uncertain events such as collisions or unintended grasping movements of the human, those not expected to end in a handover. Under these circumstances the robot should be able to keep itself and the object safe, i.e. the hand should not be damaged nor should the object fall. This is an extremely complex problem which involves quickly readjusting the fingers to maintain the stable grasping during a potentially large perturbation. Moreover, a multi-fingered grasp may not be manipulable, i.e. the object cannot be moved by the robot hand in some directions, yet forces during an unintended handover could force the object to move in that direction, causing damage to the robot and resulting in the object falling. Instead of trying to maintain the current grasp, the hand could move to a different configuration to achieve another stable grasp. That would entail a contact loss with the object at some point in time and a high chance of the object dropping. In summary, if the object is perturbed by external forces which should not result in an object handover, maintaining a stable grasp could turn out not to be possible. However, in the following sections we present a control system that was empirically found to keep the object grasped while perturbations act on the object, and an algorithm that successfully detects events that should lead to the robot releasing the object.

\section{A. Grasping effort controller}

The starting assumption in our approach is that the object is grasped in a stable configuration ready for the handover. Since the experimental platform used is the Shadow hand we use three of the five fingers (endowed with BioTAC tactile sensors) to manually set a hand configuration that holds the object (see Section III). We index the three fingers used as $j=1,2,3$, where $j=1$ is the thumb, $j=2$ is the first finger, and $j=3$ is the middle finger. Given the configuration of each finger joints $\mathbf{q}_{j}$, and the corresponding torques measured $\boldsymbol{\Gamma}_{j}$, the wrench we set on the hand for the stable grasping can be obtained in the robot base reference as ${ }^{B} \overline{\mathbf{F}}_{j}=J_{j}\left(\mathbf{q}_{j}\right)^{\dagger} \boldsymbol{\Gamma}_{j}$, where $J_{j}\left(\mathbf{q}_{j}\right)^{\dagger}$ is the pseudo-inverse of the Jacobian for finger $j$, and the superscript $B$ states the wrench is in the base reference. Converting these forces and torques to the object reference frame, jointly with the friction coefficients, and the normals at the contact points, provides the necessary conditions to maintain a stable grasp. The straightforward technique to maintain the stability of the grasp in the presence of a perturbation would be to maintain the wrenches in the object reference frame ${ }^{O} \overline{\mathbf{F}}_{j}$

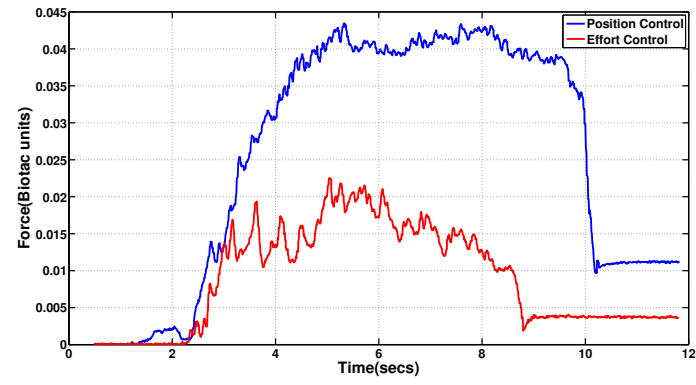

Fig. 1. Changes in the fingertip force length for effort vs. position control

constant, provided the palm reference suffers only small changes. Obviously, trying to maintain the configuration of the fingers through a position control will result in large commanded torques to the joints, potentially damaging the hand and dropping the object.

Instead of controlling the wrenches in the object reference frame, we propose to maintain and restore the contact forces and torques as computed in the base frame ${ }^{B} \overline{\mathbf{F}}_{i}$ for a stable grasp for each finger. The stable grasp wrench ${ }^{B} \overline{\mathbf{F}}_{i}$ is used as a reference in an effort joint control where the perturbed measured wrench ${ }^{B} \mathbf{F}_{j}$ is fed back to the controller. Considering the fingers individually, and given the difference between the $j$-th finger contact wrench ${ }^{B} \mathbf{F}_{j}$ and the one for the stable grasp we apply the effort $\boldsymbol{\Gamma}_{j}=$ $K_{j} J_{j}\left(\mathbf{q}_{j}\right)^{T}\left({ }^{B} \overline{\mathbf{F}}_{j}-{ }^{B} \mathbf{F}_{j}\right)$, where $J_{j}\left(\mathbf{q}_{j}\right)$ is the Jacobian of finger $j$ at the joint position $\mathbf{q}_{j}$, and $K_{j}$ is a square gain matrix of size equal to the joints to control. As an example of the behaviour of this controller, if a perturbation increases the contact force while maintaining the direction and torque, the finger will move backwards to keep the force constant. In general for a perturbed wrench the product with the Jacobian produces the motion of the finger to compensate from the external forces and torques. Since the grasped object is rigid, all three fingers perceive a change in the contact forces and torques and they will move individually to maintain the stable grasping wrench in the base frame. Although the motion can have a different effect on the wrenches in the object reference frame, because the friction of the rubber fingertips of the BioTAC is large, we experimentally found that this control mechanism kept a stable grasp while the object moved due to external perturbations. This control mechanism implements compliance in the tactile force.

For a fixed position control system in the fingers a force perturbation would results in a contact loss or increased efforts in the joints, which in the case of the Shadow hand could result in broken tendons. To illustrate the effect of the force increase when controlling the position of the fingers we performed an experiment where a perturbation force was applied to a single finger. Figure 1 shows the time evolution of the norm of the forces sensed in a finger using the BioTAC (see Section II-B for how the force is computed) with a position control vs. our proposed controller. Despite the Shadow hand fingers providing some compliance through 
its mechanical design with tendons and springs, the force sensed for a small perturbation is more than twice the force sensed when the effort controller is running, reducing the risk of damaging the hand.

\section{B. Cartesian force estimation using the BioTAC}

Provided the hand joints have torque sensors attached, the wrench applied to the object can be easily computed from the measured torques $\boldsymbol{\Gamma}$ using the pseudo-inverse Jacobian matrix for the corresponding configuration $J(\mathbf{q})^{\dagger}$ as ${ }^{B} \mathbf{F}=$ $J(\theta)^{\dagger} \boldsymbol{\Gamma}$. However, the sensors included in the joints of the Shadow hand measure the tension difference between the tendons [9], not the applied torque in the corresponding joint. We rely on the SynTouch BioTAC [10] tactile sensors installed on each finger holding the object to estimate the contact force in the fingertips ${ }^{B} \mathbf{f}$, instead of the applied wrench. The BioTAC sensor provides a variety of sensing modalities from which the contact force can be estimated; specifically it can be computed using the pressure and skin deformation. Upon contact, the increase in pressure measured by the BioTAC $(P)$ can be converted into the norm of a contact force $(|\mathbf{f}|)$ by simply using the relation $|\mathbf{f}|=P a$, where $a$ is the contact area with the object. While the pressure can be directly obtained from the sensor, the contact area needs to be estimated from the impedance measured by the 19 electrodes located across the finger core. The impedance is directly related to the distance between the core and the rubber skin at their corresponding locations, therefore a decrease in the impedance from the resting level (when there is no contact) corresponds to a deformation on the rubber skin. In our previous work [11] we presented an approach to compute the contact area using the electrodes, by approximating the contact area corresponding to each electrode $i$ as a circle of radius $r_{i}$ equal to half the distance between the electrode and its nearest neighbour. The total contact area of the fingertip can be obtained as a weighted average of these individual areas:

$$
a=\sum_{i} \lambda_{i} \pi r_{i}^{2}
$$

where $\lambda_{i} \in[0,1]$ is a scale factor. The scale factor $\lambda_{i}$ is a piece-wise linear function of the change of the impedance value $\bar{e}_{i}$ of each electrode relative to the resting level, such that at the resting level (or above) $\lambda_{i}$ is zero (meaning no contact at the electrode position), and it linearly increases to 1 for decreasing impedances up to a minimum threshold $e_{m}$ (in our case fixed at $e_{m}=-400$ ). Finally, $\lambda_{i}$ is 1 for values below the threshold.

Although the fingertip of the BioTAC also applies a torque at the contact point, there is no way to estimate it, or compute the component of the force tangential to the object. We must assume, therefore, the full length of the force is applied in the direction perpendicular to the contact surface. In [12] a technique is presented to estimate the contact direction based on the unit vectors normal to the BioTAC fingertip at each electrode position. The basic idea is similar to the one used here to compute the area, i.e. to obtain a weighted average of the normal vectors using the change in the corresponding impedances relative to the resting levels. Given the normal vectors for the electrodes of the BioTAC $\hat{\mathbf{n}}_{i}, i=1, \cdots, 19$, the total estimate of the contact force is computed as:

$$
\mathbf{f}=\frac{|\mathbf{f}|}{\left|\sum_{i} \lambda_{i} \hat{\mathbf{n}}_{i}\right|} \sum_{i} \lambda_{i} \hat{\mathbf{n}}_{i}
$$

where $\lambda_{i}$ and the force norm $|f|$ are defined above. Finally, because the force is computed in the reference frame of the fingertip, Equation 2 corresponds to ${ }^{E} \mathbf{f}$, that we convert to the base reference frame in order to use it as a valid feedback signal for the effort controller, while the torques at each contact point are considered to be zero.

\section{Object Handover and release detection}

Existing works implement handover controllers based on the evidence that humans rely on the changes in the load force to control grasping forces. Moreover, humans complete the handover procedure when the person delivering the object feels that the receiver is pulling from the object [7]. We designed our handover release algorithm based on these two events: the change in the perceived load force, and the issue of a pulling force by the receiver. However, in our case the detection of these events becomes more complex because of the interaction with the effort controller that deals with the potential perturbation forces. The estimate of the load force in the base frame ${ }^{B} \mathbf{f}_{L}$ corresponds to the sum of all the contact forces ${ }^{B} \mathbf{f}_{L}=\sum_{j}\left({ }^{B} \mathbf{f}_{j}\right)$, and when an external action is being carried out over the object there is a change in the norm of ${ }^{B} \mathbf{f}_{L}, \Delta^{B} \mathbf{f}_{L}$. This norm changing event triggers a recursive Bayesian estimation process to identify the type of event, and only when the perturbation force sensed by the fingers (compensated by the effort controller) points in the outward direction of the palm, the robot releases the object. The detection of the specific event is performed through the angular deviations of the forces in the three fingers in the fingertip frame, we will denote ${ }^{E} \mathbf{f}_{j}$ for finger $j$, with respect to the unperturbed forces $E_{\mathbf{f}_{j}}$. Algorithm 1 shows the proposed approach for reliable object handover, which works on top of the effort controller presented in Section II$\mathrm{A}$ and is described in the subsections below.

1) Event detection: At every time step, a fixed size sliding window of duration $\Delta t$ seconds $(\Delta t=0.05)$ is updated to include the latest forces ${ }^{B} \mathbf{f}$ measured by the BioTAC. We divide the window in two equal size sequences ${ }^{B} \mathcal{W}^{1}$ and ${ }^{B} \mathcal{W}^{2}$, where ${ }^{B} \mathcal{W}^{1}$ denotes the oldest data and ${ }^{B} \mathcal{W}^{2}$ the most recent. To reduce the noise from the BioTAC in the force estimate, the variation on the load forces is computed as $\Delta^{B} f_{L}=\left|E\left[{ }^{B} \mathcal{W}^{1}\right]-E\left[{ }^{B} \mathcal{W}^{1}\right]\right|$, where $E\left[{ }^{B} \mathcal{W}^{i}\right]$ denotes the expected value of the corresponding sub-window ${ }^{B} \mathcal{W}^{i}$. If this value goes above some fixed threshold we can determine that an external force is acting on the object. We chose the threshold $\Delta f_{t h}$ experimentally from data collected while perturbing the object with the effort controller running, since selecting the value without the control system generates a threshold that is too large. The general event detection 


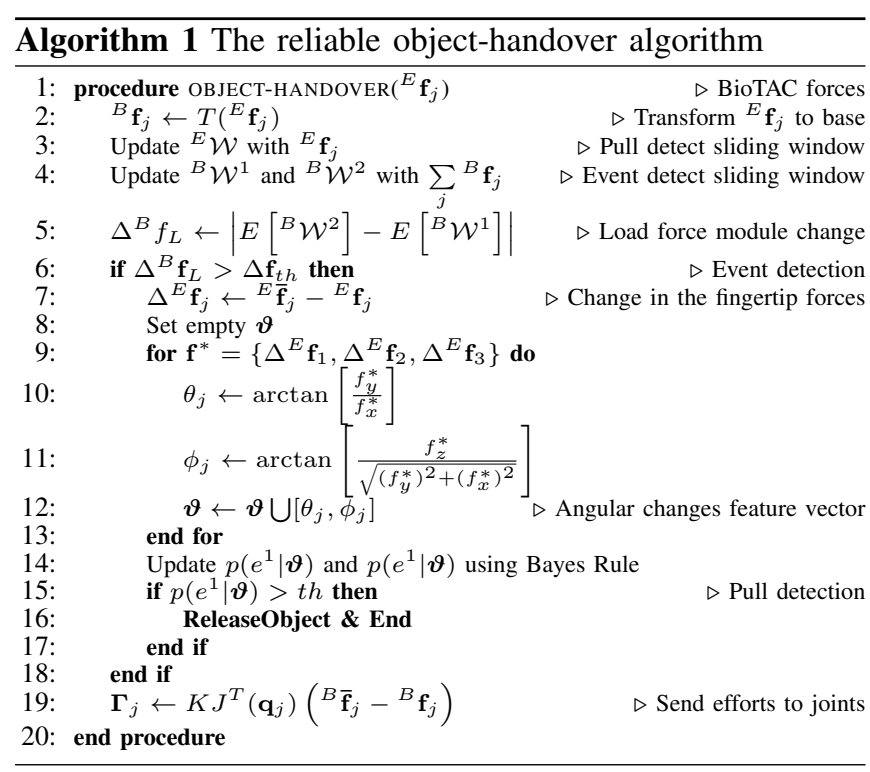

corresponds to line 6 in the algorithm and triggers the event classification.

2) Release detection: Although the variations in the load force $\Delta^{B} f_{L}$ are good indications for event detection, we experimentally found that the force without reference frame changes ${ }^{E} \mathbf{f}_{j}$ are better suited to discriminate the direction of the perturbation force. Therefore, in order to detect when the object is being pulled by the human, we have modelled the variations of the contact force for each individual finger $\left({ }^{E} \mathbf{f}_{1},{ }^{E} \mathbf{f}_{2}\right.$, and $\left.{ }^{E} \mathbf{f}_{3}\right)$ with respect to the resting position forces ${ }^{E} \overline{\mathbf{f}}_{j}$. Similar to the event detection phase, at every time step, a window ${ }^{E} \mathcal{W}$ of $\Delta t$ seconds is updated with the latest estimates of ${ }^{E} \mathbf{f}_{j}$ provided by the BioTAC. We use the deviation of the forces $\Delta^{E} \mathbf{f}_{j}$ in the three fingers to detect whether the perturbation is caused by a pulling event and compute ${ }^{E} \mathbf{f}_{j}=E\left[{ }^{E} \mathcal{W}\right]$ as the expected value of the sequence ${ }^{E} \mathcal{W}$. We define a feature vector containing the azimuth and elevation angles $\left(\theta_{j}\right.$ and $\left.\phi_{j}\right)$ of each $\Delta^{E} \mathbf{f}_{j}$, $\boldsymbol{\vartheta}=\left\{\theta_{1}, \phi_{1}, \theta_{2}, \phi_{2}, \theta_{3}, \phi_{3}\right\}$, computed from the Cartesian coordinates of these force changes in the fingertip frame. If we denote $\mathcal{E}=\left\{e^{1}, e^{2}\right\}$ the discrete events to be identified, i.e. pulling the object and any other event, we can obtain training sets from the robot running the effort controller to approximate the conditional probability of the feature vectors $\boldsymbol{\vartheta}$ given the events $e^{1}$ and $e^{2}, p\left(\boldsymbol{\vartheta} \mid e^{b}\right)$. We modelled these probabilities as mixtures of Gaussian distributions, of the form:

$$
p\left(\boldsymbol{\vartheta} \mid e^{b}\right)=\sum_{i=1}^{K_{b}} \alpha_{i}^{b} \mathcal{N}\left(\mu_{i}^{b}, \Sigma_{i}^{b}\right)
$$

where $K_{b}$ is the number of Gaussians in the mixture for event $e^{b}, \alpha_{i}^{b}$ is the weight of the $i$-th Gaussian, and $\mathcal{N}(\mu, \Sigma)$ represents a normal distribution with mean $\mu$ and covariance $\Sigma$. While we used the Expectation-Maximisation (EM) algorithm to estimate the parameters of the mixture model, the number of Gaussian functions was chosen based on the increase of the likelihood of the data given the parameters of the model. We found the optimal number of Gaussians for our experimental data was 4 for the pulling event and 5 for all other actions, i.e. pushing in different directions and rotating the object.

Having these conditional probabilities $p\left(\boldsymbol{\vartheta} \mid e^{b}\right)$ for each event, and given a new feature vector $\vartheta$ we tried to classify the events using a Maximum Likelihood approach. Although it can successfully detect pulling events, we noticed a high number of false positive classifications. Because of the sequential nature of the problem, we implemented instead a Recursive Bayesian Estimation approach which reduced the frequency with which false positives appeared. The initial event probability was distributed evenly among both possible events, i.e. $p\left(e_{1}\right)=p\left(e_{2}\right)=\frac{1}{2}$, while in subsequent estimates we use the latest posterior probabilities of each event as prior for the next prediction. Therefore, when a new feature vector $\vartheta$ is computed, we re-compute the probabilities for each event $e^{b}$ as:

$$
p\left(e^{b} \mid \boldsymbol{\vartheta}\right)=\frac{p\left(\boldsymbol{\vartheta} \mid e^{b}\right) p\left(e^{b}\right)}{p(\boldsymbol{\vartheta})}
$$

where $p\left(e^{b}\right)$ is actually the posterior probability in the previous iteration, and $p\left(\boldsymbol{\vartheta} \mid e^{b}\right)$ is given by the GMM model of the corresponding event $e^{b}$. Updating the probabilities of the two events, our system can reliably distinguish the pulling event $e^{1}$ by comparing $p\left(\boldsymbol{\vartheta} \mid e^{1}\right)$ with a fixed threshold value th adjusted empirically. This corresponds to line 15 of Algorithm 1 and results in the arm executing a fixed command to release the object.

\section{EXPERIMENTAL RESULTS}

As mentioned earlier our experimental setup consists on a Shadow Robot Hand with Syntouch BioTAC tactile sensors installed in the thumb, first and middle fingers. We manually set an initial configuration of the fingers in the centre of the hand workspace and recorded the joint positions as a starting point to set the grasping for all the experiments. Selecting this position allowed large individual finger motions due to the disturbances without lost of contact. From the initial approximate position, the fingers were manually adjusted to generate a stable grasp of the object, generating slightly different grasp configuration in terms of forces for each experiment. Before the experiments were performed the electrodes of the BioTAC were calibrated, to avoid drifts on the readings due to changes in the sensor gel after a series of runs. Figure 2 shows the configuration of the hand for a series of experiments performed with different object shapes.

\section{A. Force adaptation while holding}

Our first set of experiments was designed to evaluate the response of the effort controller to maintain stable grasping of the object in the presence of external perturbations (cf. Section II-A). The hand grasped three foam objects with different geometric shapes (cube, cylinder and triangle) and used the initial grasping forces as a reference for the controller. Then we tested different perturbing forces (pushing 


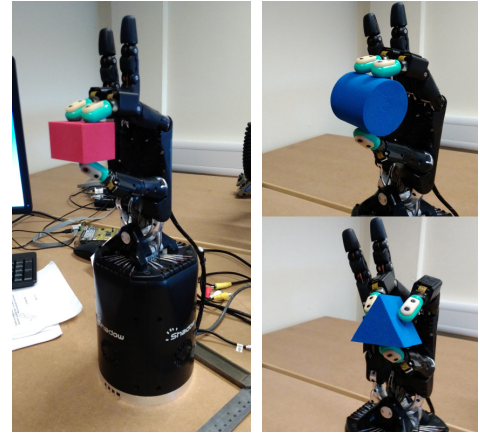

Fig. 2. Experimental set-up

up and down, rotating, and pulling and pushing the object) to evaluate the response of the proposed approach. It is worth noting that at this point the object handover algorithm was not being tested, so the hand had to adapt also to pulling forces. Despite fingers adapting individually, and the controller having no information on the geometry of the object, we found in our experiments that the system keeps contact with the object maintaining a stable grasp in different configurations. Figure 3 shows the time evolution of the components of the contact forces in the base frame for each finger when an external perturbation is applied to generate a clockwise rotation. The constant horizontal line represents the reference to the controller, i.e. the initial grasping forces, while the solid vertical lines correspond to the beginning and end of the perturbation over the object. As the external forces are applied, the fingers change their position and the contact forces deviate from the reference, while trying to keep the difference as small as possible. Once the perturbation is removed, the controller keeps trying to restore the reference contact forces but since the configuration of the fingers has changed the hand cannot generate the exact same forces. It is worth noting at this point that each finger has only three joints after the palm, so the grasping configurations are not manipulable; however the controller maintains the forces close to the initial reference.

\section{B. Force adaptation and object handover}

We also performed pulling detection experiments combined with the effort adaptation controller to evaluate the reliability of the handover mechanism. We tested the response of our approach to a variety of consecutive events (the same disturbances applied in the previous section) including at the end a pull force that had to result in a handover, i.e. the hand opens the fingers releasing the object. Figure 4 shows some images of a sequence of movements performed during one of the experiments, where the object was rotated and then pulled from the robot hand, i.e. signaling a handover event. During the rotation movement the hand adapted to keep the contact forces close to the initial grasping forces (see Figures 4(b) and $4(\mathrm{c})$ ), while the object was released when the pulling force was detected, Figures 4(d) to 4(f). We evaluated the algorithm five times for each perturbing force (two opposite rotations, lateral forces and pushing) followed by a pulling
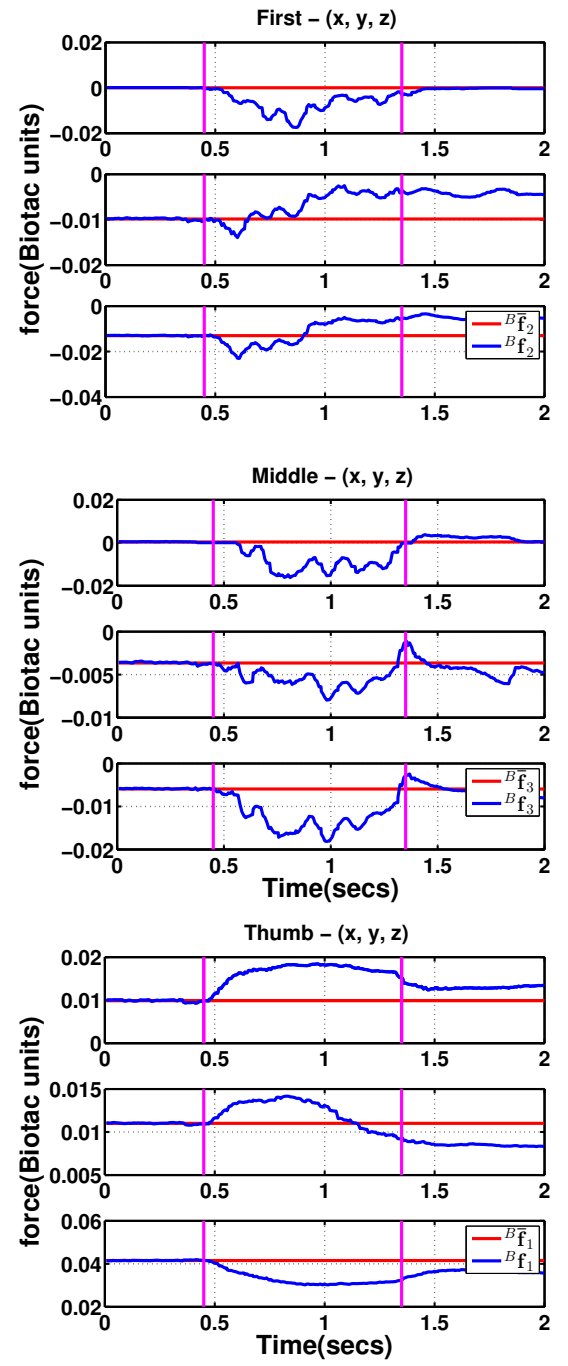

Fig. 3. Effort controller response against object rotation event (Experiment 1)

force (see attached video). Figure 5 shows the time evolution of the components of the forces in their corresponding endeffector frame for the above experiment for each finger. The first two solid vertical lines in the time sequence represent the start and end of the perturbation leading to the object rotation, while the final vertical line signals the pulling force and consequent object handover. Although the configuration of the hand changes after the first perturbation, our approach successfully detects the pulling event and proceeds to release the object.

\section{CONClusions AND FUTURE WORK}

This paper proposes a reliable object handover algorithm combining an effort joint control and the detection of pulling events, and presents its implementation on a Shadow Robot Hand endowed with BioTAC tactile sensor. While previous works assume the interaction between the robot and the human should result in an object handover, this paper considers the situations where that might not be the intention or the outcome. Our experimental results show the fingers 


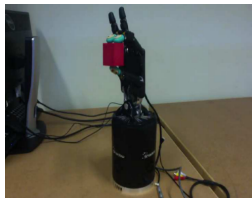

(a)

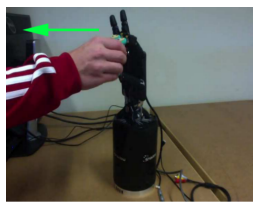

(d)

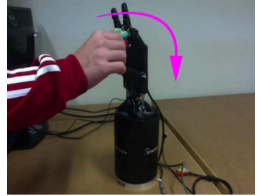

(b)

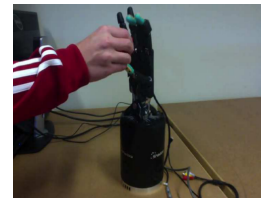

(e)

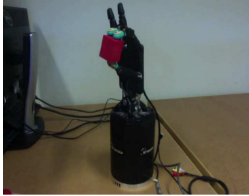

(c)

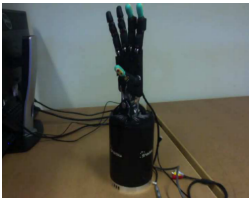

(f)
Fig. 4. Object rotation and pulling event sequence (Experiment 2)

can adapt to the disturbances from the initial stable grasping situation, while managing to continue to hold the object in new configurations. We also show that pulling the object (as a precursor to handover) can be differentiated from other forces that should not result in the robot handing the object over. Our algorithm works only with the tactile estimates of the contact force instead of using the contact wrench, which cannot be computed from the Shadow Robot hand joint torque sensors.

Although the implemented effort controller successfully adapts to new configurations in the presence of perturbations, the limited degrees of freedom of the fingers relative to the palm restrict the object movement. In the case of the Shadow Robot hand this means the point of contact between the fingers and the object changes slightly, potentially affecting the stability of the grasp. Moreover, we noticed that the detection of the pulling event is highly sensitive to the geometry of the object that the hand is grasping. This problem could be solved through a better Gaussian mixture model for pull detection or by the inclusion of visual information about the geometry of the object. Using vision would enable a richer interaction between the robot and the human creating the opportunity to investigate reliable human to robot object handover.

\section{REFERENCES}

[1] M. Cakmak, S. Srinivasa, M. Lee, J. Forlizzi, and S. Kiesler, "Human preferences for robot-human hand-over configurations," in Proceedings of the IEEE/RSJ 2011 Interntional Conference on Intelligent Robots and Systems, 2011.

[2] W. Chan, Y. Kaiuchi, K. Okada, and M. Inaba, "Determining proper grasp configurations for handovers through observation of object movement patterns and inter-object interaction during usage," in Proceedings of the 2014 IEEE/RSJ International Conference on Intelligent Robots and Systems, 2014, pp. 1355-1360.

[3] K. Nagata, Y. Oosaki, M. Makikura, and H. Tsukune, "Delivery by hand between human and robot based on fingertip force-torque information," in Proceedings of the 1998 IEEE/RSJ International Conference on Intelligent Robots and Systems, 1998, pp. 750-757.

[4] A. Edsinger and C. Kemp, "Human-robot interaction for cooperative manipulation: Hnading objects to one another," in Proceedings of the 2007 Symposium on Robot and Human Interactive Communicaiton (RO-MAN), 2007.

[5] J. Bohren, R. Rusu, E. Jones, E. Marder-Eppstein, C. Pantofaru, M. Wise, L. Mösenlechner, W. Meeusen, and S. Holzer, "Towards
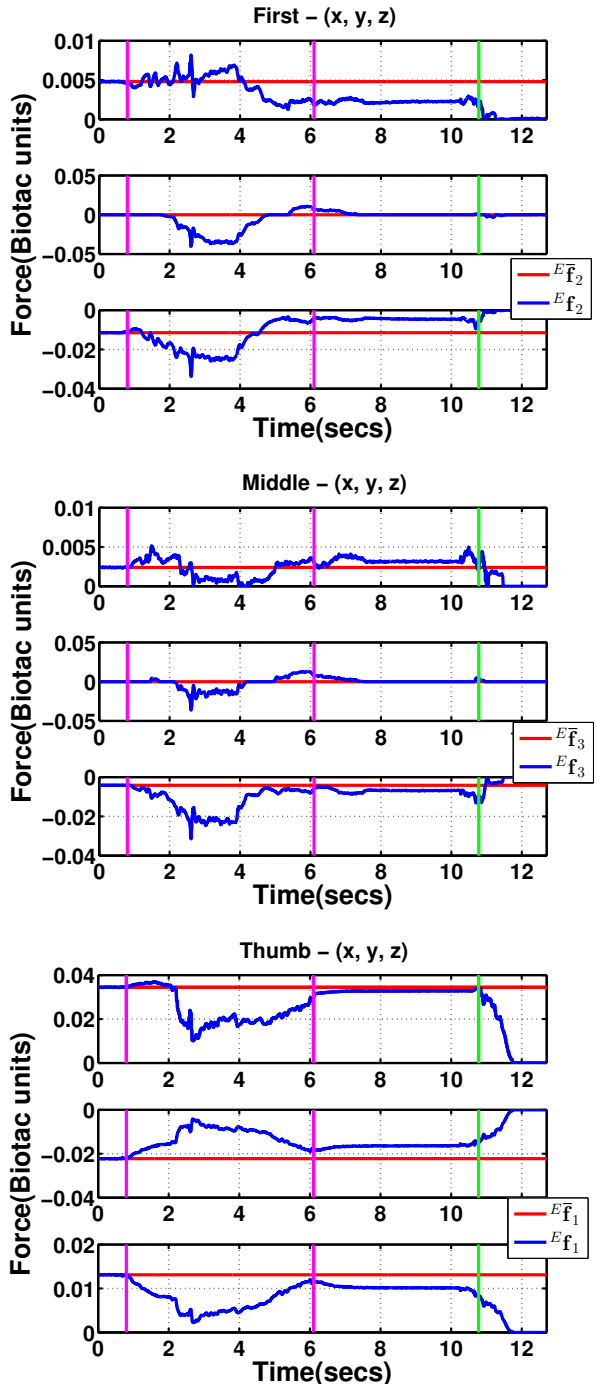

Fig. 5. Forces response against object rotation and pulling events (Experiment 2)

autonomous robotic butlers: Lessons learned with the pr2," in Proceedings of the 2011 IEEE International Conference on Robotics and Automation, 2011, pp. 5568-5575.

[6] I. Kim and H. Inooka, "Hand-over of an object between human and robot," in IEEE International Workshop on robot and Human Communication, 1992, pp. 199-203.

[7] W. P. Chan, C. A. Parker, H. F. Van der Loos, and E. A. Croft, "Grip forces and load forces in handovers: implications for designing humanrobot handover controllers," in In Proceedings of the seventh annual ACM/IEEE international conference on Human-Robot Interaction, vol. 6, March 2012, pp. 9-16.

[8] W. Chan, C. Parker, H. V. der Loos, and E. Croft, "A humaninspired object handover controller," International Journal on Robotics Research, vol. 32, no. 8, pp. 971-983, 2013.

[9] H. Elias, EtherCAT Dual CAN Hand User Manual, v0.9 ed., Shadow Robot Company.

[10] J. Fishel, G. Lin, and G. Loeb, "Syntouch LLC biotac product manual, v. 16," Tech. Rep., 2013.

[11] A. G. Eguíluz, I. Raño, S. Coleman, and T. McGinnity, "A multi-modal approach to continuous material identification through tactile sensing," in International Conference on Intelligent Robots and Systems (IROS 2016), 102016

[12] Z. Su, J. Fishel, T. Yamamoto, and G. Loeb, "Use of tactile feedback to control exploratory movements to characterize object compliance," Frontiers in Neurorobotics, vol. 6, p. 7, 2012. 